\title{
LA PROCEDENCIA DE LOS REPO- BLADORES EN EL REPARTIMIENTO DE ORIHUELA
}

Por

JUAN TORRES FONTES

FRANCISCO VEAS ARTESEROS 
The present article is concernd with the place of origin of those people who, in search of new lands in which to settle and better prospects for the future, took advantage of excellent conditions offered by the king of Castille and came to Orihucla, where the lands were to be distributed. A study is made of toponymic surnames, which clearly reveal the place of origin, and of first names which also give and idea, though less precise, of the likely provenance. It must be admitted that what is offered is the first part of a study which, until further material has been fully investigated, must remain at an embryonic level.

Even so, it is possible to distinguish an abundance of CatalanAragonese compared with the number of settlers from the Crown of Castille, and a large remainder of people whose geographical identity is hard to determine $i$ fwe take the land register as our sole source of information. 
En 1243 se firma el tratado de Alcaraz por el infante don Alfonso, como heredero de Castilla y en nombre de su padre Fernando III, con los enviados del rey lbn Hud de Murcia, y los representantes de los senores que se mantenían independientes de su soberanía, entre los que la Crónica General enumera a los de Crevillente, Alicante, Orihuela, Elche, Aledo, Cieza, Ricote y otros lugares uque eran señoreados sobre si»; clara manifestación de la división política existente en el reino murciano.

De conformidad con este acuerdo, la hueste real mandada por don Alfonso, se dirige al reino de Murcia, en donde iba a entrar sin obstáculo alguno para llegar a la capital el día 1 de Mayo. Seguidamente se hizo cargo del alcázar y dispuso la instalación de guarniciones militares en las principales fortalezas de los lugares y villas que se le habian entregado sin resistencia. Pero también hubo rebeldía de algunas plazas, lo que obligaría a disponer su conquista mediante la acción militar. Mula, Orihuela y Lorca se sometieron; la primera sin condiciones y las otras dos acabaron aceptando el pacto de Alcaraz en el año 1244. Mas todavía tuvo que esperar don Alfonso a contar con medios propicios, entre ellos una escuadra, para culminar la ocupación de la totalidad del reino con la conquista de Cartagena, en la primavera del año siguiente.

De esta forma la incorporación del reino de Murcia a la Corona de Castilla no se produce de manera uniforme, pues si bien en su mayor parte goza del estatuto de protectorado y sólo les afecta directamente la 
soberanfa castellana en cuanto a tener que abonar parte de las rentas pertenecientes a la realeza, admisión de guarniciones en las fortalezas y pérdida de las propiedades de los huidos, en otras villas y comarcas, como Mula y Cartagena, conquistadas sin capitulación, la ocupación castellana fue inmediata, lo que supuso la expulsión de los musulmanes del interior de los recintos urbanos y la pérdida de sus bienes.

Ya entonces a Castilla se le presenta un problema insoluble, cual era la falta de medios humanos, tanto para sustituir al musulmán que emigra como para asegurar con abundante número de vecinos cristianos un reino periférico como el murciano, frontero con Aragón y Granda y con un litoral marftimo expuesto de forma permanente a las depredaciones de los corsarios. Problema que se mantiene en situación no extremadamente grave durante algunos años.

Con el transcurso del tiempo las cosas comienzan a cambiar, y ya rey de Castilla Alfonso X empieza a preocuparse del futuro de la demarcación murciana, porque, casi de inmediato, surgen otras cuestiones, si bien la principal era la vecindad del reino de Aragón, que permanentemente mostrará un sordo propósito de rectificar acuerdos, ya que su expansión habia sido contenida precisamente hacia el reino de Murcia por el tratado de Almizra, firmado en 1244, por el que no sólo se reconocía la soberania castellana sobre el territorio murciano, ratificando as! lo acordado en Cazola, sino que suponia también la desaparición de la frontera aragonesa con los musulmanes, siempre útil como fuente de ganancias y por insólitas amistades.

La estancia de Alfonso X en el reino de Murcia en los años 1254 y 1257 supone la introducción de innovaciones y una intervención cada vez mayor, sobresaliendo entre las muchas manifestaciones que se producen la sustitución del merino mayor por un adelantado, la desaparición de la intitulación del reyezuelo musulmán en la emisión de moneda y el asentamiento de cristianos en tierras que concede el monarca perteneciente al cillero real, procedentes de los musulmanes huidos o por la adquisición que se hace a los islámicos con permiso de sus autoridades. 
Esta presencia castellana, que también se manifiesta en las ciudades donde continua existiendo mayoría musulmana, significa una lenta y gradual intromisión que origina exigencias o excesos que quebrantan las condiciones de seguridad en que hasta entonces hablan vivido los musulmanes, y se inicia entonces una pausada pero mantenida emigración hacia Granada y Africa, comenzada por aquellos que más podían perder, afanosos de conservar sus bienes, que venden sus propiedades o las abandonan; así como sabios, alfaquies, filósofos, poetas y una rica burguesía que busca en su precavido exilio encontrar lugar apropiado para continuar sus negocios, sin las apreturas y precarias condiciones que cabría esperar de una precipitada hurda. $Y$ los que no se marcharon estuvieron siempre atentos a las promesas que al-Alhamar de Granada les hacía llegar, asegurándoles su ayuda militar y la de los africanos, para que tanto Andalucía como el reino de Murcia recobrasen su libertad.

Se produce entonces la sublevación mudéjar de Andalucía y Murcia en 1264, que no se termina de sofocar en el reino murciano hasta dos años después, merced a la generosa ayuda de Jaime I y de sus huestes aragonesas. Pero la guerra traería consigo unas consecuencias difíciles de evitar. Por una parte la sublevación dejaba sin efectividad las capitulaciones otorgadas por los castellanos entre 1243 y 1245; y por otra son muchos los musulmanes que perecen o son apresados y reducidos a la esclavitud, otros murieron mientras emigraban y muchos también los que buscaron en la huida la posibilidad de conservar su libertad. No es posible cuantificar esta baja numérica de los mudéjares murcianos, pero el desarrollo de los acontecimientos y sus consecuencias posteriores muestran que debió alcanzar cifras muy considerables.

Tres testimonios lo ponen de manifiesto. En los repartimientos de Lorca y Orihuela no existe la menor reserva territorial para los musulmanes, si se exceptua, en ei segundo, las condiciones favorables que se conceden al «rais» de Crevillente. En Murcia, por la capitulación concedida por Jaime I, después modificada por Alfonso X, se les cede la mitad de la huerta y campo de su jurisdicción asi como el arrabal de la 
Arrixaca. Ni Alfonso el Sabio pudo mantener mucho tiempo estas condiciones, ni el repartimiento de Murcia se pudo acabar por falta de pobladores para establecer y trabajar las tierras menos ricas y de mayor coste.

Todo lo anteriormente dicho produce una doble consecuencia: continuidad de la emigración musulmana y parcial vuelta a sus regiones de origen de muchos pobladores cristianos, que habían acudido esperanzados a la repoblación del reino, en la creencia de que todo iba a resultar fácil y beneficioso. Ambos casos conducen a un mismo resultado, que no es otro sino la despoblación de amplias comarcas, abandono del ámbito rural, concentración urbana y aumento alarmante de las expediciones o aceifas de zenetes y granadinos quc, aprovechando la despoblación, recorren el reino en todas direcciones con el único objetivo de obtener el mayor número de cautivos y ganados.

\section{LA REPOBLACION}

Sería Murcia el foco más atractivo para los ilusionados pobladores que acudieron a la llamada de los repartimientos: huerta, mayor territorio, capital del reino; todo, en suma, se ofrecía atrayente. Sin embargo, el contingente humano que Castilla podía aportar era escaso, ya que Andalucía absorbra y necesitaba todo el excedente y aún algo más de lo que el reino castellano estaba en condiciones de proporcionar. Por otra parte cuenta, y mucho, la vecindad aragonesa y, especialmente, que han quedado atrás las repoblaciones de Mallorca y Valencia, y son muchas las gentes de Cataluña, Aragón y Navarra que buscan en la aventura de la emigración las esperanzas y posibilidades que podía proporcionarles el Sureste. Y, por encima de todo, el hecho de que Jaime I propiciara el asentamiento de todos aquellos integrantes de sus tropas que quisieran permanecer en las tierras que ellos mismos habían liberado.

Se conjugan de esta manera dos factores: la falta de población, causada por la baja considerable de musulmanes que se hacía sentir en todas 
partes, agravada por el hecho de que muchos castellanos, venidos a primera hora, también se marcharon a sus anteriores lugares de residencia o a otros en busca de mejores perspectivas; y falta de potencial humano que se amortigua parcialmente con la aportación aragonesa, y el compromiso del monarca aragonés de heredar a los numerosos contingentes de catalano-aragoneses que habian integrado su hueste circunstancialmente y que, terminadas las operaciones bélicas, soñaban con la posesión de tierras mejores que las de sus núcleos de origen; lo que se pone de relieve cuando la crónica castellana de Fernando IV, al comentar la invasión aragonesa dirigida por Jaime II y la sorprendente facilidad con que la realiza, dice que lo hizo por consejo de los de la tierra, que eran catalanes "mdieronse todas las villas e los castillos, salvo ende Lorca, que moraban castellanos, e otrosi, Alcala e Mula....).

Jaime I, por su parte, en su Llibre manifiesta: «poblam hi be $\mathrm{X}$ milia homnes d'arms entre de nostra terra e daltres..J. El número parece excesivo y la exageración es viento favorable que tiende a elevar las cifras de los cronistas. Cascales, que al parecer también sabía contar, nos proporciona unas cifras que bien pudieran ser muy cercanas a las de la realidad: Murcia 2.500, Lorca 600, Orihuela 1.000 y Mula 80; en total unos 4.180, cifra que queda muy lejos de los diez mil de don Jaime y mucho más aceptable por cuanto coincide con las cifras que nos proporcionan las consultas de los respectivos repartimientos.

También a la hora de la conquista se plantea la disyuntiva de como asegurar el reino, conocida la escasez de medios humanos, así como también la necesidad de mantener la continuidad de los cultivos. Jaime I entendía que lo más conveniente era establecer y heredar a cien caballeros de sus huestes, vasallos cristianos y moros, dueños de señoríos y villas, un tanto a la manera que se mantenían en el bajo Aragón o en zonas valencianas. Alfonso X, por el contrario, era partidario de promocionar los núcleos urbanos, creando poderosos concejos con amplios términos, y efectuar minuciosos repartos de las mejores tierras entre el mayor número posible de pobladores, con preponderancia de caballeros y arte- 
sanos; a su lado señoríos de no mucha extensión, con la salvedad del otorgado a su hermano el infante don Manuel, y donaciones a las Ordenes Militares. Consideraba el rey Sabio que la conjunción de esta diversidad de personas sería suficiente para proporcionar la seguridad apetecida al adelantamiento y el que no faltase la producción agrícola y la ganadería para su normal abastecimiento.

\section{EL REPARTIMIENTO DE ORIHUELA: PROCEDENCIA DE LOS REPOBLADORES}

El códice titulado Llibre dels Repartiments recoge un total de seis particiones, aunque dentro de esta denominación genérica entran por igual revisiones y ampliaciones de los dos primeros repartimientos, y se extienden cronológicamente desde 1266 a 1314.

Dentro del aspecto social cabe destacar cuatro grupos de repobladores. Mención especial tienen los que participan en la defensa del castillo y villa cuando en 1264 se produce la sublevación mudéjar, que obtienen beneficios realmente extraordinarios. También los donadios, otra minoría privilegiada que obtiene espléndidas concesiones, en las que predominan caballeros y adalides cuya reiterada estancia en las particiones parece indicativa de la necesidad de su presencia. Las categorías normales: minoría de nobles y caballeros que obtienen amplios beneficios pero sin el resultado apetecido, ya que en su mayor parte los venderán y partirán del suelo oriolano, o pierden sus concesiones por ausencia. La Iglesia y las Ordenes Militares obtienen no muchos beneficios y en condiciones muy semejantes a las otorgadas a los demás pobladores, si bien es cierto que la Orden de Santiago recibe el dominio sobre el señorío de Algorfa, segregado del repartimiento. Y, finalmente, el grupo más numeroso en el que también se establecen distinciones como sucede con las viudas, a quienes por su peculiar situación se atiende muy solícitamente.

Un estudio completo que respondiera al título del presente trabajo, necesitaría un análisis pormenorizado de todos los repartimientos así 
como de las revisiones y rectificaciones que de los mismos se llevan a efecto, objetivo de momento imposible de realizar, no sólo porque desbordaría los límites propios de un artículo, sino especialmente por la dificultad que supone el poder precisar certeramente la procedencia de muchos de los pobladores, trabajo que requiere un estudio al detalle de cada uno de ellos. Así pues, para la elaboración del presente artículo nos hemos basado en la totalidad del Libro de los Repartimientos, sin distinguir los repartimientos propiamente dichos de sus revisiones, eliminando los nombres repetidos cuando de la misma persona se trata.

Es nuestro propósito, por tanto, dar ahora un avance sobre la procedencia de todos aquellos que acudieron a Orihuela a la hora de los repartimientos deduciéndola de su onomástica, lo que ha planteado no pocas dificuitades, entre las que se encuentran la aparición del nombre solamente o bien acompañado del oficio o apodo; apellidos comunes extraordinariamente diff́ciles de determinar; patronímicos que responden a la adopción del nombre paterno; nombre seguido del topónimo sin estar precedido de la preposición de, lo que si bien permite intuir su origen no sucede lo mismo cuando de situarlos en un punto concreto se trata; o bien por la forma en que se han escrito sus nombres, apellidos u oficios, a veces mal copiados y que pueden inducir a error.

Con frecuencia se expresa con claridad meridiana la procedencia de los repobladores (Juan de Belchite, Bernat de Argensola, Simón de Játiva, Fernando de Alicante, Pedro Pérez de Lorca, Miguel de Toro), otras veces solamente de una forma indirecta (Arnalt Barcelon, Jacob Sabadell, Pedro Alicante), y más abundantemente el modo en que aparecen escritos los nombres ha servido para su ubicación regional (Bernat, Arnalt, Berenguer, Jacme, Martin, Gutier, Juan). Pero, a pesar de todo, ha quedado un buen número de pobladores que por la forma en que aparecen inscritos es imposible determinar su procedencia, existiendo la posibilidad de que una investigación más detenida permita reducir esa cifra de gentes venidas al repartimiento oriolano que calificamos como indeterminados. 
Para elaborar los cuadros que a continuación desarrollaremos se ha partido del reino que, a tenor de nuestras investigaciones, aportó un mayor contingente de pobladores, para pasar después a aquellos cuyo suministro fue menor. Siguiendo esta premisa, la Corona de Aragón aparece en primer lugar con 648 pobladores, seguida de Castilla, Navarra y ya en mucho menor número aquellos que proceden de países extranejros. Hemos de señalar que para clarificar los cuadros se ha recurrido a la tradicional división provincial, por lo que hay que hacer constar que la actual provincia de Alicante se encontraba entonces en su mayor parte inmersa dentro del reino de Murcia, así pues solamente aquellas poblaciones alicantinas situadas al Norte del puerto seco de Biar se han incluido en la Corona de Aragón, apareciendo el resto en el gráfico correspondiente a Castilla. Para una mejor comprensión se han dividido los repobladores en aragoneses (catalanes, aragoneses y valencianos) y no aragoneses (castellanos, navarros y extranjeros).

Adelantamos ahora el resultado estadístico de nuestros estudios en los siguientes cuadros, con el tanto por ciento respecto al total:

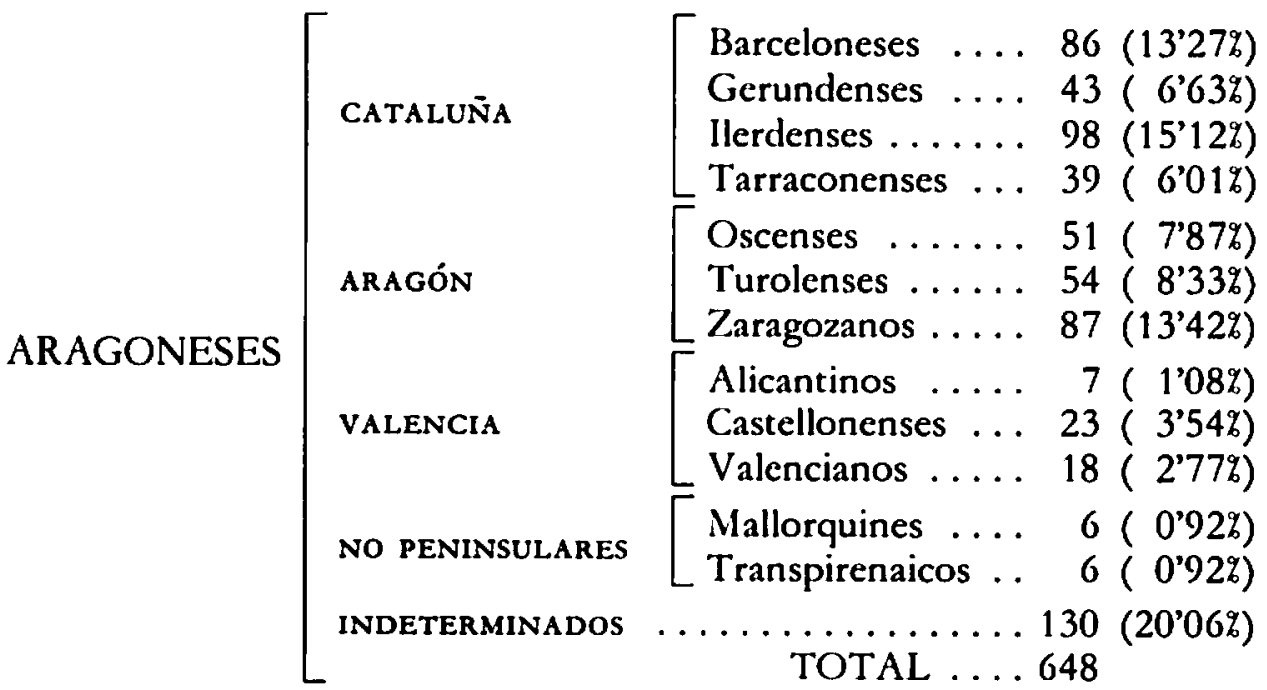


Dentro del grupo catalán destaca por su contribución numérica al poblamiento oriolano Lérida y su actual provincia, que con 98 es la que más aporta. De lugares como Limiñana, Vall-Llebrera, Balaguer, y en menor proporción Basella, Camarasa, Campmajor, Isona, Tárrega, la misma capital y de otros muchos partieron un buen número de gentes, pudiéndose fijar el origen de una buena parte de ellos en las comarcas de Tremp, Sort, Seo de Urgel y la propia Lérida.

Los oriundos de Barcelona constituyen también un grupo importante, destacando entre ellos los procedentes de la capital y su amplia comarca: Berga, Berta, Canet de Mar, Ca'n Bou, Manresa, Masquefa, Marfa, Montpalau, Moya, Sabadell, San Feliu, Molins, Vic, San Pol, etc. Un tercer grupo está constituido por los Ilegados desde Gerona, cuya procedencia está muy repartida situándose a la cabeza Ripoll, seguida de Freixe, Farell y Gerona capital, partiendo de las diversas localidades de la comarca gerundense en número menor. Finalmente el grupo menos representativo dentro de los catalanes es el procedente de Tarragona, en donde los originarios de Flix son los más numerosos, mientras que de otras poblaciones como Montblanch, Robiols, Riufret, San Jaime, Soldevila y otras proviene una cantidad mucho más reducida.

El segundo bloque lo conforman aquellos repobladores procedentes del núcleo aragonés en el que sobresalen los zaragozanos, siendo Ejea, Zaragoza, Daroca, Sos, Pina y Siurana las que aportan un mayor contingente poblacional, procediendo el resto de núcleos de población como Añón, Ariza, Artieda, Belchite, Calatayud, Luesia, Mallen, Maluenda, Tarazona y otros. Oscenses y turolenses concurren en número similar a las tierras levantinas; dentro de los primeros el grupo más numeroso procede de la comarca jacetana siendo de Jaca el contingente más nutrido, seguida por Huesca, Berdún, Rocafort y Argovieso. Desde Teruel capital procede la mayor cuantía de repobladores, siendo con mucho la que más contribuye al poblamiento oriolano, ya que Alcañiz, Albarracin, Corbalán, etc, despiden un número modesto de sus vecinos. 
El reino de Valencia es, dentro de los aragoneses, el que menos pobladores proporciona, un total de 48 que se distribuyen casi por igual entre las actuales provincias de Castellón y Valencia, y, en menor proporción, Alicante, englobado entonces en una mínima parte de su extensión en el reino valenciano. De la comarca de La Plana proceden 23, siendo Segorbe y Morella las que encabezan cuantitativamente la representación castellonense, mientras que Castellón, Burriana, Segarra y Onda, entre otras, mantienen grandes diferencias respecto a las dos primeras. De Valencia, conquistada por Jaime I en tiempo no muy lejano, no van a trasladarse muchos a Orihucla, siendo la igualdad la característica general, puesto que de Onteniente, Valencia, Oliva, Castelfabib, Bocairente y otras saldrán muy pocos pobladores. Caso similar al del Alicante aragonés, en donde Cocentaina proporciona los más pobladores.

En los no peninsulares hemos encuadrado a los pertenecientes a aquellos territorios foráneos pero que formaban parte de los Estados de la Corona de Aragón; su concurrencia es ciertamente escasa y coincidente en lo referente a la cantidad. Procedentes de Mallorca vienen a Orihuela cinco pobladores de los que dos son de la capital, mientras que de Ibiza sólo llega uno. Caso similar es el de los denominados ultrapirenaicos, originarios del Rosellón y de Cerdaña, siendo Montpellier el lugar desde donde acude más gente; y el resto de Carcasona, Monfort y Perpignan.

El último bloque lo constituyen los indeterminados, denominación bajo la cual hemos agrupado a todos aquellos pobladores difíciles de encuadrar en una comarca determinada. Dificultad que se ve aumentada porque el códice que contiene los repartimientos no es el original, sino un traslado del Siglo XIV, cuando Orihuela era ya territorio aragonés, lo que se pone de relieve en una manifiesta catalanización de nombres realizada por el escribano, que no en vano era aragonés. Se encuentran incluidos en este grupo, entre otros, los repobladores de nombres, apellidos $u$ oficios que denotan una procedencia catalano-aragonesa como los siguientes: Belsoler, Benages, Bestreta, Boceta, Brançon, Caminada, Cap- 
gali, Carcyyl, Cortell, Cremades, Cuch, Dauza, Fuset, Bernat, Gres, Guerra, Laxar, March, Marchs, Morlach, Paracge, Puig, Puigviro, Puigzivoro, Tegores, Torrella, Torrell, Viach, Villanueva, Villauta y otros muchos; además de los indicados aquellos iniciados con «ça, çes, za, na, nes, des, dez, den», tales como çaplana, despla, desclerge, despont, çesprats, etc. Es indudable que dentro de este grupo se encuentran incluidos muchos catalanes, pero también es cierto que hay otros pertenecientes a Aragón, Valencia, algunos mallorquines y pocos ultrapirenaicos, existiendo además la posibilidad de que se hallen varios navarros e incluso castellanos a los que se les catalaniza el nombre.

Mayores dificultades hemos tenido para localizar geograficamente a los castellanos y navarros, pues si bien en unos casos se expresa su procedencia (gallegos, sorianos, salmantinos, conquenses, toledanos, andaluces, vascos, pamploneses, estelleses, etc.), en la mayoría de los que se han inventariado ha sido necesario recurrir a la forma castellana de los nombres, apellidos u oficios (Domínguez, López, Martínez, Pérez, Rodríguez, Gómez, Sancho, Rui, Ruiz, Díaz, Núñez, Lara, Tello y otros), por lo que son muy abundantes los que conforman el bloque de indeterminados dentro de los no aragoneses.

No sucede los mismo con los de procedencia extranjera, realmente muy pocos, pues sus lugares de origen se expresan con claridad meridiana y no hay lugar a errores en cuanto a su localización geográfica se refiere.

En el cuadro siguiente se expresan las cantidades de repobladores no aragoneses con su porcentaje respecto al total: 


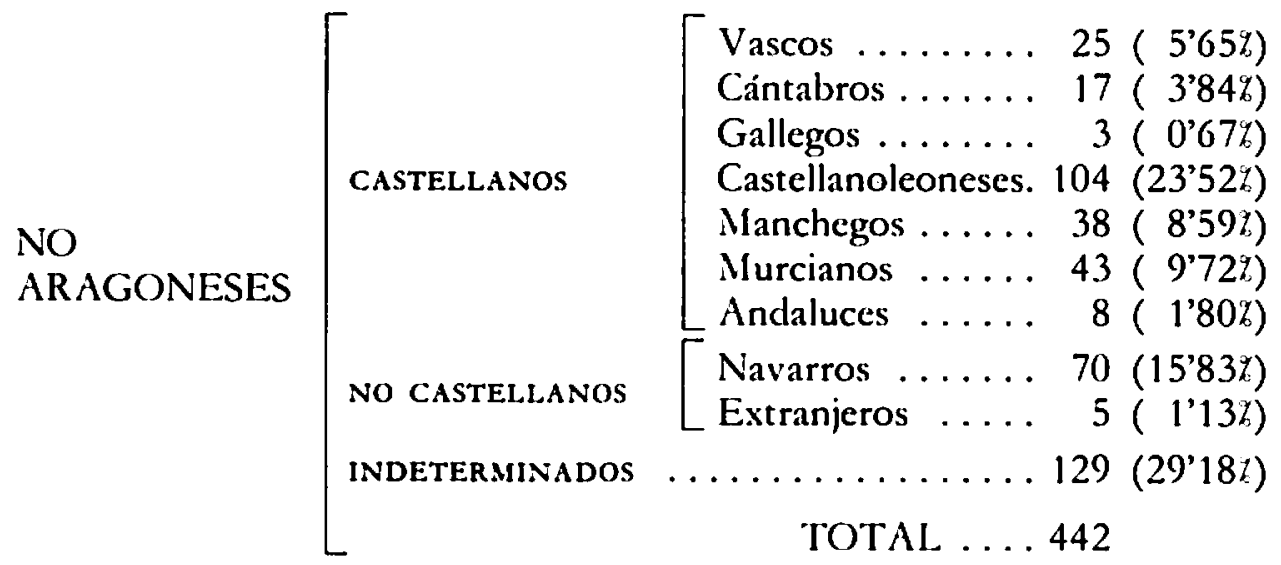

Vascos, cántabros y gallegos aportan al repartimiento de Orihuela un total de 45 pobladores, que en su mayoría proceden de la comarca santanderina, seguida por Guipúzcoa y en menor medida las restantes hasta llegar a Galicia, desde donde proceden tres individuos. Núcleos de población como Aguero, Villar de Salcedo, Vergara, Ayala, Carnedo, Santander, Santiago y otros verán como varios de sus moradores se alejaban en busca de las prometedoras posibilidades que se les of recían en el Sureste.

Dentro de los no aragoneses la mayor proporción numérica corresponde a los castellanoleoneses. Su cifra es importante si contemplamos el cómputo global de castellanos que, a tenor del cuadro, acuden a Orihucla que es de 238 , pero es una aportación irrisoria si se compara con la extensión territorial del reino de Castilla, lo que es una prueba más del gran problema de escasez de pobladores que sufría la Corona castellana, al que antes hemos aludido.

Burgos, «cabeça de Castilla», como la denominan tantas veces los documentos reales, se encuentra al frente de los repobladores castellanoleo- 
neses presentes en Orihuela, seguida por los procedentes de las comarcas de Logroño y Soria, mientras que de las otras circunscripciones viene menos gente. Burgos, Aranda, Miranda, Contreras, Mena, Medrano, Santo Domingo de la Calzada, Almazán, Deza, Soria, Jubera, San Esteban de Gormaz, Molina de Aragón, Hinojosa, Sigüenza, Ayllón, Palencia, Astudillo, Valladolid, Mahide, San Justo, Toro, Folgoso, Alcántara, Consuegra, Mora, Toledo y muchos otros lugares constituyen los focos de procedencia de estos repobladores. Mención especial merece Toledo, de donde llegan un buen número de nobles que obtienen buenos dominios señoriales en el repartimiento oriolano.

Dentro del quinto grupo sobresalen por su numerosa participación los albacetenses y conquenses. Los primeros con un alto porcentaje de Alcaraz, que con cinco es la que más representación tiene; en este sentido no hay que olvidar que los de Alcaraz tuvieron una activa participación contra los mudéjares sublevados en 1265 , tomando la villa y castillo de Cartagena, por lo que su presencia en Orihuela está más que justificada. Cuenca, repoblada por Alfonso VIII y muy próxima a Aragón, remitirá el contingente más numeroso, un total de 26 individuos, procedentes de Alarcón, Huete, Belmonte, Villalvilla, Paracuellos, Uclés, Cuenca e Hito, todas con buena participación.

El reino de Murcia aporta 43 pobladores, de los cuales 29 proceden de lugares actualmente enclavados dentro de la provincia de Alicante: Agost, Almoradi, Castalla, Catral, Elche, Nompot (antual Monforte), Villena, Sax, Jijona y otros. A la vez que desde Abanilla, Caravaca, Cartagena, Lorca y Mula también acuden a la demarcación oriolana gentes en busca de tierras en donde asentarse, pretendiendo alejarse un poco de la línea fronteriza con Granada.

Los andaluces son muy escasos y los procedentes de Estepa, Mairena, Sevilla, Ubeda y pocos lugares más son lo que hacen acto de presencia en Orihuela. 
Además de los castellanos al repartimiento de Orihuela van a acudir grupos de pobladores procedentes del reino de Navarra, que en su mayorfa porceden de Cascante, Tudela, Aibar, Azagra, Estella, Falces, Fustiñana, Monteagudo, Murriones, Olite, Pamplona y en menor cantidad de las otras poblaciones de la de demarcación navarra. Hemos podido precisar una concentración en lo que se refiere al origen de los repobladores navarros situada en las comarcas de Cascante, Tudela, Aibar y Azagra, lugares que aportan un mayor contingente.

Los escasos extranjeros que acuden atraidos por los repartimientos proceden de Florencia dos, Avignon uno, y de Flandes y Hungria uno respectivamente, siendo procedente de este último país uno de los acompañantes de la reina doña Violante. En total cinco son los representantes de otros países europeos en la demarcación oriolana, destacando la presencia del judío florentino Jacob Felipe, activo comerciante del Siglo XIII, que debía buscar en Orihuela nuevos caminos para continuar con sus negocios.

Finalmente, en el bloque de los indeterminados hemos incluido a los castellanos y navarros de dudosa localización, ya que existen contradicciones al encontrarnos con nombres catalanes seguidos de un topónimo castellano, lo que nos induce a pensar que esa catalanización de nombres, a la que antes nos hemos referido, es más que una suposición una realidad. También se aprecian muchos gallegos, lo que no quiere decir que viniesen de las lejanas tierras galaicas, sino que nos inclinamos a pensar que eran descendientes de gallegos afincados en otras partes del reino castellano, que conservan el apellido toponímico de sus progenitores.

He aqui el resumen estadístico de todos los pobladores de Orihuela, con su porcentaje respecto al número total: 


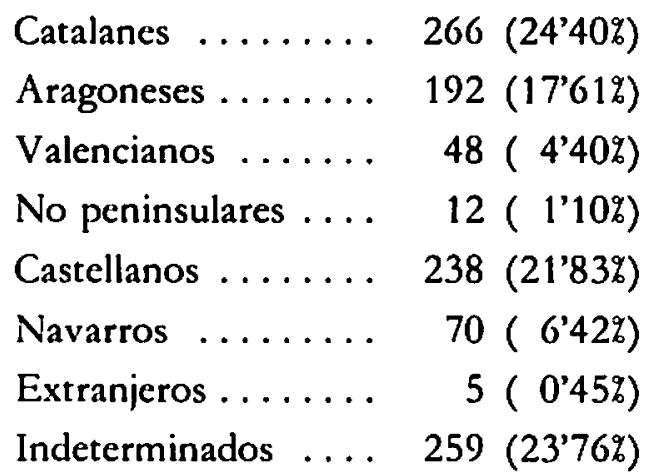

¿Qué consecuencias pueden deducirse de esta larga exégesis estadística?. Ante todo una mayor presencia de catalanoaragoneses, entre los cuales los catalanes representan el mayor porcentaje con el 24 ' $40 \%$ del total de repobladores, mientras que los castellanos, con el $21^{\prime} 83 \%$, guardan una gran diferencia con respecto al grupo catalanoaragonés. Pero los indeterminados, que representan un alto porcentaje, podrán variar los resultados aquí inicialmente expuestos, una vez que se profundice más en su estudio y nuestras investigaciones nos permitan precisar mucho más su procedencia, así como delimitar hasta qué punto se convierten en catalanas, gracias a la pluma del escribano, nombres que pueden serlo castellanos, si bien la aportación de la Corona de Aragón siempre creemos que será mayor. 